\title{
ESTIMATING FUTURE DEMAND FOR HOSPITAL EMERGENCY SERVICES AT THE REGIONAL LEVEL
}

\author{
Bożena Mielczarek \\ Institute for Industrial Engineering and Management \\ Wrocław University of Technology \\ Wrocław, POLAND
}

\begin{abstract}
The level of demand for hospital emergency services is closely connected to the demographic characteristics of a region's population. The objective of this study is to examine the influence of changes in population size and structure on the volume of emergency service needs exhibited by patients arriving at hospital emergency departments in the area. The Monte Carlo simulation model examines demographic trends at the regional level, formulates forecasts for population changes, and extrapolates the simulated patterns of the demand for acute services. The model includes data on the population in 9 districts that surround Wrocław, the capital of Lower Silesia (Poland) and data on acute visits to emergency departments located in the region. Our analysis suggests that two age groups, i.e., children aged 0-4 and elderly people aged 60 and over, are responsible for a large share of the changes in the demand level.
\end{abstract}

\section{INTRODUCTION}

The Polish emergency medical service system (EMS) includes pre-hospital medical care, which is provided by medical emergency teams, and hospital/ambulatory care, which is provided by hospital emergency departments (EDs). An ED performs diagnostic and therapeutic services and serves as the point of admission for most patients with acute medical conditions. Local governments are obliged to deliver emergency services to the citizens of their regions. Contracts between the purchaser (the National Health Fund NFZ) and the providers are conditional on the forecasted demand for acute services and the cost of relevant medical treatment in EDs. The preferences of the patients with respect to their selection of an ED are important factors during the contracting process. The prediction formula for the expected volume of emergency patients is valuable at the regional and the unit levels. At the macro level, the formula supports strategic planning decisions that are related to resource allocation to cover the demand for EMS services. At the micro level, the formula is used in the internal hospital financial planning process. For the contract values to be reliable and accurate, it is important that the tool forecasts future demand in relation to the entire region and each ED located in the region. The financial resources at NFZ's disposal are divided based on contracts with the EDs to cover the demand for hospital emergency services in the region. If emergency admissions could be forecasted accurately, contracts could cover the real costs of emergency treatment in units, and equity in access to emergency care could be assured for a region's population.

Typically, the quantitative methods applied to study demand are time series analysis methods. The most common approaches are based on the decomposition of time series and causal modeling (cf. Batal et al. 2001; Channouf et al. 2007; Champion et al. 2007). Certain authors (Setzler et al. 2009) have suggested the use of artificial neural networks in formulating emergency call forecasts for both time and location. In this paper, the regression model and the Monte Carlo (MC) simulation approach are proposed to investigate demographic trends across the region and examine the influence of changes in the population on the 


\section{Mielczarek}

volume of emergency service needs expressed by the patients arriving at hospital emergency departments in the area.

\section{LITERATURE REVIEW}

The literature on predicting EMS service demand has evolved in two primary directions. One class of models seeks methods for forecasting how the demand for ED services changes over time. The aim of these models is to deliver to EMS managers reliable information that can influence micro-level planning and facilitate the allocation of human and physical ED resources. Typically, such studies focus on the local ED, and the process of model development and validation is based on historical data while assuming that future demand will behave in a similar manner. These models are used to make short-term predictions for hourly, daily, or monthly hospital emergency arrivals. The applied methods are usually time series analysis methods. The second class of models may be described as upper-level models that predict overall spatial demand for large geographic areas over long periods (e.g., a year) using demographic and socio-economic characteristics. These models support strategic decisions, such as resource use planning, which includes the determination of vehicle placement strategies. The models are useful when making decisions regarding healthcare system expansion or reorganization. Additionally, the models could be useful when estimating future costs of the provision of EMS services when beginning contract negotiations with regional providers.

An interesting review in the EMS demand volume forecasting literature was provided by Setzler et al. (2009). Beginning with the 1970s, the authors tracked forecast models and described numerous examples of applied forecasting techniques. Batal et al. (2001) matched the daily patient volume with calendar and weather variables and applied linear regression analysis. Channouf et al. (2007) estimated the arrival rate during day-long and hour-long periods using time series models. Champion et al. (2007) used the exponential smoothing and Box-Jenkins methods to forecast the number of patients arriving daily at the hospital emergency department for the next five months. Ong et al. (2009) applied a time series analysis to describe the time-demand patterns at a local EMS unit. The authors observed that emergency attendance appeared to correspond to circadian rhythms. Jones et al. (2008) evaluated several statistical methods for predicting daily ED patients volumes at multiple, diverse hospital EDs and confirmed that daily demand for emergency services occurred in seasonal and weekly patterns. McCarthy et al. (2008) proved that demand for ED services was effectively approximated by the Poisson model, which meant that the number of arrivals that occur within the space of one hour was independent of the number that occur within the next hour. Boyle et al. (2012) conducted studies to predict the number of ED presentations and subsequent admissions on any given day of the year for a range of urban and regional hospitals. The models considered included multiple regression, autoregressive integrated moving average and exponential smoothing models. Six time series models were developed by Diaz-Hierro et al. (2012), who concluded that the simultaneous use of two models improves short-term planning. Diaz et al. (2001) studied the relations between emergency hospital admissions and environmental variables and found significant associations. The studies confirm that demand in the ED is cyclical and that the daily number of ED patients changes according to the day of the week and the time of year. The time series methods are successful in predicting the average daily number of patients. The limitation of these models is that they do not consider the possibility of sudden changes in population demographics and unmet demand.

Initial attempts to develop upper-level models of the demand for emergency services were made in the 1970s by Aldrich et al. (1971) and Kvalseth and Deems (1979). These authors estimated the number and type of calls for publicly dispatched ambulances using multivariate linear statistical models. They found that the demand for public ambulances appeared to be highly predictable and that the most important variables in terms of their effect on total EMS demand were acreage, family economic status, the rate of male unemployment and racial composition. Kamenetzky et al. (1982) estimated the demand for emergency transportation services using four independent variables: population, employment, and two in- 


\section{Mielczarek}

dicators of socio-economic status. They obtained reliable estimates and determined that the use of such a model enables planners to estimate unmet needs.

The primary factors that influence the level of demand for hospital emergency services are as follows: population demographics (i.e., age, gender, and racial characteristics), the region's socio-economic profile (i.e., employment, the number and location of healthcare providers, and housing and land use), temporal factors (i.e., hour-of-day, day-of-week, type-of-day, season, and calendar year), weather factors (i.e., temperature and precipitation), patient factors (i.e., age, gender, and triage level) and environmental factors (i.e., air pollution). Most authors have considered age-associated patterns and reached a range of conclusions. McConnel and Wilson (1998) and Lowthian et al. (2011a) found that demand for EMS services increased exponentially with age, and Chu et al. (2009) observed a relative decrease in ED attendance by patients 65 and older across certain triage groups. Interestingly, Lowthian et al. (2011b) proved that although demand for ambulances was growing faster that can be explained by population growth, it was possible to attribute only $25 \%$ of the EMS calls to ageing and population growth. Most of the studies confirm that the observed trends are geographically related and that local population demographics trends may be of importance.

In this paper, the MC simulation approach is proposed to examine the influence of demographic changes on the volume of emergency calls as represented of patients arriving at hospital emergency departments in the area. According to Mielczarek and Uziałko-Mydlikowska (2012a), MC is the preferred modeling technique for analyzing epidemiology, health promotion and health policy issues. The advantage of a simulation model is its ability to test any modification to fully understand the problem and to estimate the variability involved in the observed process. Simulation models operate on probabilistic distributions that are in most cases derived from historical datasets. A model simulates hundreds or thousands of potential scenarios and produces forecasted outputs, typically in the form of relevant means, probabilities and the dispersion of results around the expected value. To the best of the author's knowledge, no studies have been conducted that use the MC simulation approach to quantitate the relationship between the expected EMS patient volume and demographic variables. The general idea of the study was described in Mielczarek and Uziałko-Mydlikowska (2012b).

\section{PROBLEM STRUCTURING}

Healthcare in Poland is financed by obligatory health insurance contributions paid by each working citizen. The NFZ administers this income and allocates it to finance healthcare services by dividing the contributions among voivodeships according to an allocation formula. The healthcare services purchase process has been based on selective contracting between the payer (the NFZ) and the healthcare providers. Regional branches of the NFZ contract broad categories of service in respective voivodeships: primary and ambulatory care, specialized ambulatory care and inpatient care, emergency care, rehabilitation and intermediate care, long-term care, hospice and palliative care, mental healthcare and other types of care. In contrast to other healthcare services, emergency services are provided without restriction for every patient who requires medical assistance.

EMS ambulance services are funded by the state budget and administered by regional branches of the NFZ. Hospital emergency services, which are provided in admission units (AUs) and emergency wards (EWs), are reimbursed by the NFZ in accordance with daily lump-sum rates, which are defined ex ante in yearly contracts. The daily lump sum, $R$, for any AU/EW is determined in close relation to prior-year demand volume: $R=R_{S}+R_{f}$, where $R_{S}$ represents structure component and $R_{f}$ represents the function component of the daily lump sum. The $R_{S}$ is determined based on the degree at which the standard level of technical, organizational and personnel requirements is satisfied. The standard level is defined by the NFZ regional authority and published in a special announcement. The $R_{f}$ is calculated based on the number of patient visits registered during the previously reported period. 
The estimation of the next-year number and the structure of acute services to be provided in AU/EWs in the area might facilitate the determination of the fair values of contracts. If EMS admissions could be forecasted accurately, the contract would enable the real costs of emergency treatment in the unit to be covered and assure equity in access to emergency care. In this paper, the use of the regression model and the MC simulation approach is proposed for the estimation of future EMS admissions volume in the context of the entire regional demand.

The study was conducted in the Wrocław Region (WR), which is part of the Lower Silesia voivodeship in Poland. The WR encompasses 9 districts: the capital of Lower Silesia (Wrocław) and 8 districts that are close to the capital. In 2010, the WR's population comprised approximately $41.11 \%$ of the Lower Silesian population. Twelve admission units (AUs) and 5 emergency wards (EWs) operated in the area during this year. All of the AU/EWs located in the WR serve the inhabitants of the region in addition to patients from other Lower Silesia sub-regions and citizens from other Polish provinces. A patient can freely select the hospital where he or she seeks emergency care. Individuals who reside in the WR may receive emergency treatment from the AUs and EWs located in the WR and the AUs and EWs located in other Polish sub-regions.

\section{DATA AND METHOD}

\subsection{Demographic Data}

Demographic data were obtained from the Central Statistical Office (CSO 2013) webpage. The study period was 2006-2011. Independent variables were selected based on the findings described by Kamenetzky et al. (1982), who tested a range of variables and predicted EMS demand using 4 independent variables. We assumed that a model should contain a small number of variables and that the input data should be easily available from the CSO webpage. The following set of demographic statistics were considered in the model: (a) total population - according to Kamenetzky et al. (1982) population is intuitively the most important variable in determining demand; (b) two age groups (0-4 and 60+) which according to the initial analysis produced the highest percentages of EMS patients among other age groups.

\subsection{EMS Demand Data}

The data for this study were obtained from the NFZ regional branch in Wrocław (Poland). The basic datasets included information on 759,964 emergency admissions of patients residing in 9 districts in the WR. The admissions were registered in AU/EWs located in Lower Silesia. The data were provided by month for each year during the period 2006-2011. The records from the second, detailed dataset revealed 183,517 emergency visits registered in 2010 in AU/EWs located in the WR. To protect anonymity, personal data were deleted from the source files.

Table 1 provides statistics on annual emergency demand registered in all of the AU/EWs in Lower Silesia for individuals residing in the $9 \mathrm{WR}$ districts. The values describe the overall emergency needs of the WR population that were met by the emergency departments located in Lower Silesia. Data on emergency admissions to hospitals other than those in Lower Silesia were not available and are not considered here.

Table 1: Annual summary statistics on emergency demand.

\begin{tabular}{lllllll}
\hline & 2006 & 2007 & 2008 & 2009 & 2010 & 2011 \\
\hline Total population & $1,168,102$ & $1,170,067$ & $1,172,954$ & $1,177,157$ & $1,183,169$ & $1,197,462$ \\
Demand & 120,825 & 134,622 & 147,872 & 173,227 & 183,418 & 188,381 \\
Demand/population & 0.10 & 0.12 & 0.13 & 0.15 & 0.16 & 0.16 \\
\hline
\end{tabular}




\section{Mielczarek}

The numbers presented in Table 1 suggest rapid growth in EMS call volume between 2008 and 2009. However, the data are misleading, and there are at least two important reasons that explain the observable significant increase in the demand. First, the Polish healthcare system was radically transformed after 1999 when the sickness funds were established. Only three years later, in 2001, NFZ replaced sickness funds. The ongoing changes in the healthcare system (i.e., the longer and the more complicated process required to arrange specialist care) caused patients to consider hospital AUs as alternatives to general practitioner practices, which increased the number of non-emergency cases treated by the emergency system. Second, the information on the number of emergency visits to EDs is based on the historical data obtained from the NFZ registries. The monitoring and recording system used by the NFZ was unstable and the data-collection requirements were continually modified. The requirements gradually became more precisely defined, and the data transferred from hospital statistical units to NFZ regional branches became more extensive. Therefore, it was determined to forecast the future EMS demand in the WR while considering the historical data obtained from NFZ only for the period 2009-2011.

\subsection{Regression Model}

Based on the 54 data points, the regression and correlation analysis was performed. Then, using the least squares linear regression method, a model was constructed that contained only three independent variables: total population, percentage of the total population aged $0-4$, and percentage of the total population aged 60 or older. The final regression equation was as follows:

$$
D=-50069.6+0.14536 \text { Pop }+156325.5(\% \text { Pop } 60+)+412821.1(\% \text { Pop } 04) .
$$

$D$ : annual total number of emergency calls from the WR population,

$P$ : population of the WR,

$(\%$ Pop60+): percentage of the total population aged 60 or older,

(\%Pop04): percentage of the total population aged 0-4.

An adjusted $R^{2}=0.95$ and a $p$-value $<0.15$ were obtained, which indicated a good fit (Table 2).

The coefficients that precede each variable determine the number of additional patient visits (assuming that the patient is a WR resident) to ED units:

- a one-thousand person increase in the WR population increases the hospital emergency demand of the WR population by 145 ,

- an increase of $0.1 \%$ in the number of elderly people in the WR increases the hospital emergency demand of the WR population by 15,600 ,

- an increase of $0.1 \%$ in the number of children aged 0-4 in the WR increases the hospital emergency demand of the WR population by 41,300 .

Table 2: Statistics obtained with the regression analysis. Pop: population in the WR; (\%Pop60+): percentage of the total population aged 60 or older; (\%Pop 04$)$ : percentage of total population aged $0-4$.

\begin{tabular}{llll}
\hline & Coefficient & Standard error & p-value \\
\hline Intercept & $-50,069.6$ & $12,592.65$ & 0.000226 \\
Pop & 0.145356 & 0.006503 & $1.11 \mathrm{E}-27$ \\
(\%Pop60+) & $156,325.5$ & $55,807.22$ & 0.007222 \\
(\%Pop04) & $412,821.1$ & $169,067.3$ & 0.018196 \\
Adjusted $R$ square & 0.95 & & \\
Standard error & $5,940.7$ & & \\
Observations & 54 & & \\
\hline
\end{tabular}




\section{Mielczarek}

Validation was performed for the historical data that describe three demographic variables: the total population in the WR region, the percentage ratio of the elder population and the percentage ratio of children aged 0-4 for the years 2009-2011. The total quantity of demand provided by formula (1) was compared with the historical demand data collected for the WR in 2009-2011. The correlation between estimated and observed values is 0.99 , which suggests a highly consistent relationship. The output validation tests results are provided in Table 3. The observed differences indicate a good fit. Note that when the same three independent variables were included in a larger set of variables (we tested employment, unemployment, gender, and other age groups), a regression procedure selected a substantially inferior model in interpretability and goodness of fit.

Table 3: Validation of the regression model: Comparison of the estimated and actual levels of demand for emergency services in the WR.

\begin{tabular}{lllc}
\hline Year & Model demand & Actual demand & Difference \\
\hline 2011 & 179,184 & 188,381 & $-4.88 \%$ \\
2010 & 175,099 & 183,418 & $-4.54 \%$ \\
2009 & 172,273 & 173,227 & $-0.55 \%$ \\
\hline
\end{tabular}

\subsection{Monte Carlo Simulation Model}

A Monte Carlo (MC) model using an Excel spreadsheet was constructed to simulate the forecasts of the emergency hospital demand volume originating in the WR population. The key element of the MC model is the regression formula presented in (1), which links the volume of WR emergency patients to the WR population. To perform the simulation, the input probability distributions that characterize the demographic parameters of the WR must be defined. To properly model the demographic trends in the WR, the random distributions should describe the changes in the nominal values and not the nominal values themselves. The probability distributions for the annual changes of the three demographic parameters were constructed using the data published by CSO for the period 2000-2012. Table 4 presents the output statistics obtained after the fitting procedure was performed.

Table 4: Input probability distributions that describe three demographic parameters of the WR and the statistics that summarize the fitting process. SE: standard error; KE: Kolmogorov-Smirnov statistics.

\begin{tabular}{|c|c|c|c|c|}
\hline Parameter & Distribution & Parameters & Test statistics & $p$-value \\
\hline $\begin{array}{l}\text { Population growth } \\
(\triangle P o p)\end{array}$ & exponential & $\begin{array}{l}a=-1170 \\
\mu=4090\end{array}$ & $\begin{array}{l}\mathrm{SE}=0.010726 \\
\mathrm{KE} \text { test }=0.198\end{array}$ & $>0.15$ \\
\hline $\begin{array}{l}\text { Population } 60+\text { growth } \\
(\triangle P \text { op } 60+)\end{array}$ & exponential & $\begin{array}{l}a=296 \\
\mu=3950\end{array}$ & $\begin{array}{l}\mathrm{SE}=0.111233 \\
\mathrm{KE} \text { test }=0.225\end{array}$ & $>0.15$ \\
\hline $\begin{array}{l}\text { Population } 0-4 \text { growth } \\
\text { (APop04) }\end{array}$ & uniform & $\begin{array}{l}a=-1562 \\
b=3718\end{array}$ & $\begin{array}{l}\mathrm{SE}=0.14375 \\
\mathrm{KE} \text { test }=0.347\end{array}$ & $>0.15$ \\
\hline
\end{tabular}

The MC model samples the values from the three input random distributions and converts them into the form of three parameters, which are the independent variables of the regression model. The forecasted demand volume is calculated as an average value based on 500 replications. 


\section{SIMULATIONS AND COMPARISONS}

\subsection{Model Validation}

Model validation was performed for the data that describe the WR population and its structure as registered in 2009 (Pop = 1,177,157; Pop04 = 58,180; Pop60+ = 232,179) and in 2010 (Pop = 1,183,169; Pop04 $=61,316 ;$ Pop60 $+=240,643)$. The simulation experiment consisted of the following steps:

1. Sampling values that describe the changes $\triangle P o p, \triangle P o p 04$ and $\triangle P O p 60+$ that result from 3 input random distributions in accordance with the parameters in Table 4;

2. Calculating the forecasted (i.e., the next year) values for Pop, Pop04 and Pop60+; $\operatorname{Pop}(2010)=\operatorname{Pop}(2009)+\triangle P o p ;$

3. Calculating the forecasted percentage share of $\%$ Pop 04 and $\%$ Pop $60+$ in the forecasted WR population, Pop;

4. Applying regression formula (1) to predict the demand for 2010 and 2011 based on the simulated parameters Pop, \%Pop04, and \%Pop60+;

5. Replicating steps (1) - (4) 500 times and calculating the average demand value and the output statistics (Table 5).

Table 5: MC output statistics. Simulation model predictions for 2010 (2011) WR population and demand based on 2009 (2010) historical data. The \%Difference measures the percentage difference between historical values observed for 2010 (2011) and the MC model forecasts formulated for 2010 (2011).

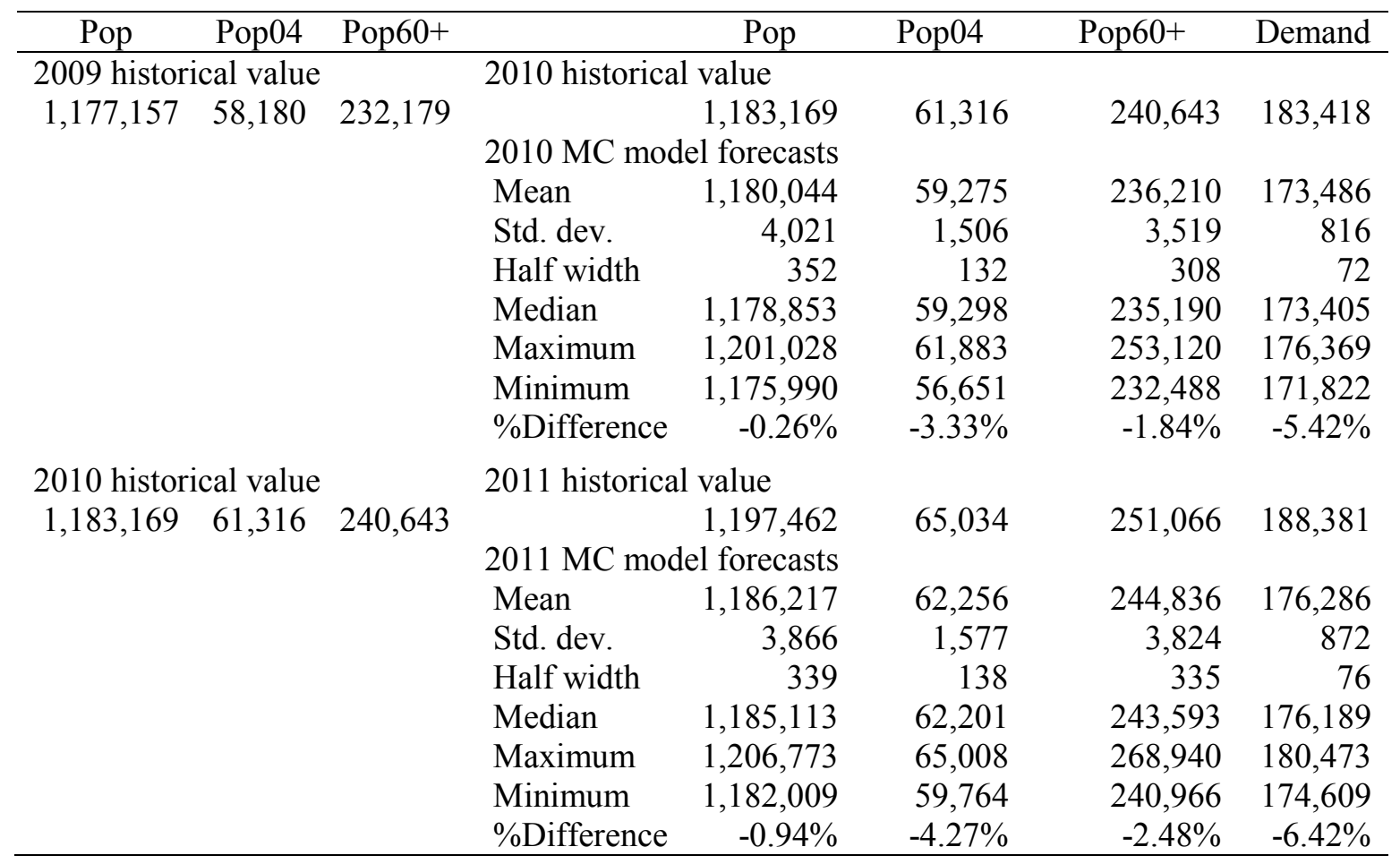

Table 5 provides descriptive statistics for the output variables obtained using the MC model. The simulation for the total population (Pop) in 2010 resulted in values slightly lower than historical $(1,180,044$ compared with $1,183,169)$. However, the difference is small $(0.26 \%$ underestimation $)$. The numbers in the two considered age groups (Pop04 and Pop60+) are also underestimated, and the percentage difference is $-3.33 \%$ and $-1.84 \%$, respectively. The prediction interval, which is defined by minimum 


\section{Mielczarek}

and maximum values collected during 500 trials, encompasses the true values in the case of all three variables. The forecasted demand level is underestimated by $5.42 \%(173,486$ compared with 183,418), and the true value falls outside the prediction interval. The mean demand exceeds the median demand, which indicates that the simulated demand values are skewed to the right. Similarly to 2010, the simulation results obtained for 2011 resulted in values that were slightly lower than historical. However, the difference remains acceptable $(6.42 \%$ of underestimation in demand values).

The underestimation of EMS demand most likely results from the specificity of the WR, which is the third largest educational center in Poland. The inflow of students from October until June increases the total population up to $12 \%$ (according to CSO) and generates the additional EMS calls served by AU/EWs in WR. The second reason that explains the lower simulation outputs compared with the historical values is connected to non-demographic factors that influence the level of demand for hospital emergency services, e.g., the region's socio-economic patient profile or environmental factors. However, considering the observed discrepancy, we may assume that the evidence supports the conclusion that a simulation model provided good forecasts for the expected level of emergency services in the WR.

\subsection{Simulation Results}

\subsubsection{Base Case Scenario}

A series of scenarios was developed to evaluate through computer simulation the influence of demographic trends in the WR on the forecasted level of demand for hospital emergency services exhibited by the WR population. The historical data on $2011 \mathrm{WR}$ demographic characteristics were input into the MC model, and the simulation was performed for 2012. The simulation predicted a $4.23 \%$ decrease in 2012 in emergency patient visits in the WR region (Table 6). Additionally, the WR population was predicted to grow by $0.24 \%$. Population growth in the two considered age groups (children age $0-4$ and people $60+$ ) was also observed.

Table 6: The MC model output statistics. The simulated changes in the WR population structure and the emergency demand level in 2012 (n/a: data not available).

\begin{tabular}{|c|c|c|c|c|}
\hline 2012: output simulation values & $\begin{array}{r}\text { Population } \\
0.24 \%\end{array}$ & $\begin{array}{r}\text { Population } 0-4 \\
1.75 \%\end{array}$ & $\begin{array}{r}\text { Population } 60+ \\
1.72 \%\end{array}$ & $\begin{array}{r}\text { Demand } \\
-4.23 \%\end{array}$ \\
\hline $\begin{array}{l}\text { 2012: values extrapolated based on the } \\
\text { incomplete CSO and NFZ data }\end{array}$ & $0.32 \%$ & $\mathrm{n} / \mathrm{a}$ & $\mathrm{n} / \mathrm{a}$ & $-3.70 \%$ \\
\hline
\end{tabular}

The incomplete data that describe the WR total population in 2012 (3 quarters of 2012, as published by CSO) and the incomplete data on emergency demand in the WR (6 months of 2012, as collected by NFZ) confirm the credibility of the simulation forecasts.

\subsubsection{Simulation Based on CSO Demographic Forecasts}

The MC simulation model was input with the data on the forecasted demographic changes in the WR. The forecasts were formulated by the CSO based on the data from 2007. According to the CSO (Table 7), the WR population will exhibit a continuous and stable increase (annually from $0.13 \%$ in 2013 to $0.07 \%$ in 2020). The number of children aged 0-4 will decrease, and the predictions indicate a stronger slope rate in the subsequent years (from $0.47 \%$ in 2013 to $-1.76 \%$ in 2020). The number of older individuals (60+) will increase. However, the growth rate will weaken in the subsequent years (from $2.92 \%$ in 2013 to $1.22 \%$ in 2020).

Assuming that the factors that drive EMS arrivals remain much the same in the future, the series of simulation experiments were performed to observe the changes in emergency demand in accordance with 


\section{Mielczarek}

the demographic trends forecasted by the CSO. Figure 1 presents the simulated percentage changes in the total number of emergency hospital presentations originating in the WR population for 2012-2020 in connection with the growth rates of three population parameters according to demography forecasts published by the CSO. It is apparent that the level of demand is continuously increasing. However, the growth is less intensive in the following years. The percentage values that describe the simulated demand growth rates are positive. However, these value decrease from $2.34 \%$ in 2012 to $0.18 \%$ in 2020 .

Table 7: CSO demographic forecasts. The predicted annual changes in the WR population.

\begin{tabular}{rrrr}
\hline & Population & Population 0-4 & Population $60+$ \\
\hline 2013 & $0.13 \%$ & $0.47 \%$ & $2.92 \%$ \\
2014 & $0.12 \%$ & $0.06 \%$ & $2.75 \%$ \\
2015 & $0.11 \%$ & $-0.29 \%$ & $2.71 \%$ \\
2016 & $0.10 \%$ & $-0.66 \%$ & $2.47 \%$ \\
2017 & $0.10 \%$ & $-0.93 \%$ & $2.24 \%$ \\
2018 & $0.08 \%$ & $-1.25 \%$ & $2.07 \%$ \\
2019 & $0.06 \%$ & $-1.50 \%$ & $1.53 \%$ \\
2020 & $0.07 \%$ & $-1.76 \%$ & $1.22 \%$ \\
\hline
\end{tabular}

Simulation experiments demonstrate that the youngest population strongly influences the estimated level of hospital emergency visits. The forecasted decrease in the number of children aged 0-4 significantly affects the EMS needs in the WR and slows the predicted demand growth rate, which - in contrast - is increased by the increasing number of individuals aged over 60. According to McConnel and Wilson (1998), those in the older population group are nearly twice as likely to generate emergency calls than younger persons. With the stable level of the entire population, these two age groups produce the observable changes in the EMS demand level.

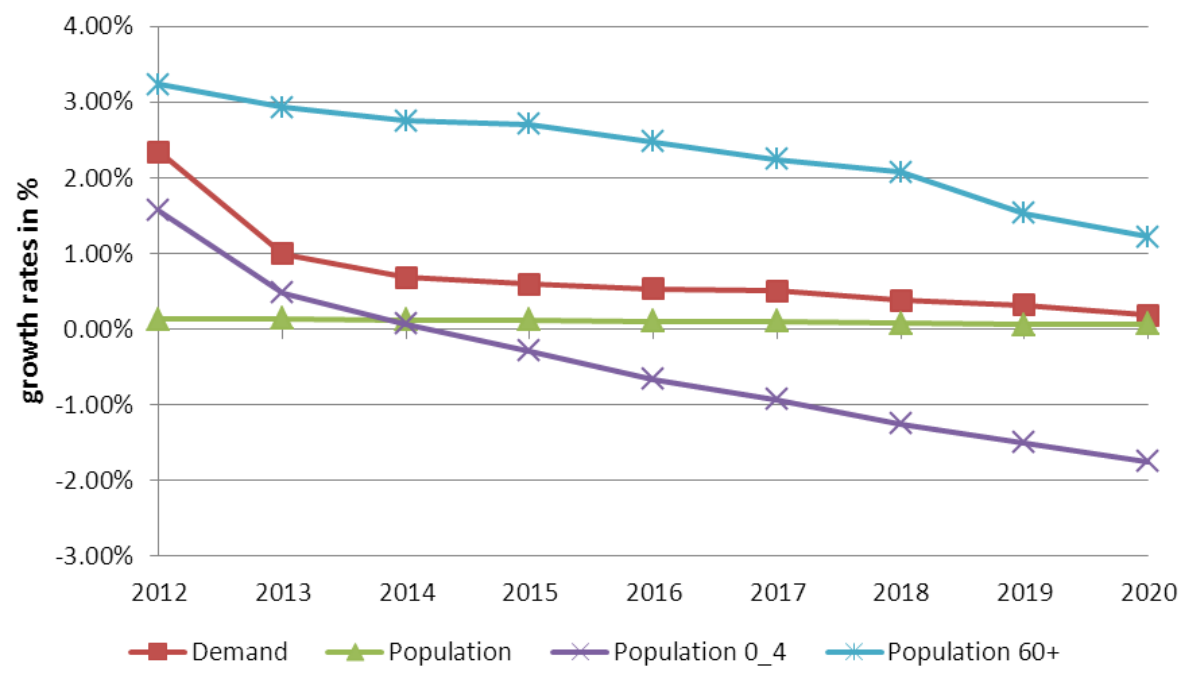

Figure 1: Simulation of WR emergency demand based on the CSO demographic forecasts. Annual growth rates in \% compared with the previous year.

\subsubsection{What-if Simulation}

We developed and tested two what-if scenarios with the initial values set as in the base case simulation. In scenario 1, we varied the growth rate of the youngest population from $-10 \%$ to $10 \%$. In scenario 2 , we changed the growth rate of the $60+$ age group from $-10 \%$ to $10 \%$. 
In scenario 1 (Figure 2), when the number of children age $0-4$ increased by $10 \%$, the EMS demand compared with the base case increased up to $0.05 \%$. The opposite trend, i.e., a decrease in the number of the youngest population, lowers the EMS demand only by $0.01 \%$ compared with the base case. The fluctuations are so small that they should be ignored. We may conclude that the slight decrease in the number of children age 0-4 will not significantly influence the estimated EMS needs. The results of scenario 2 indicate that the changes in the 60+ age group influence the total EMS demand level more strongly when increasing and when decreasing the number of individuals in this age group. The same percentage changes in the two age groups provide stronger dynamics of demand values when the changes are applied to the older population.

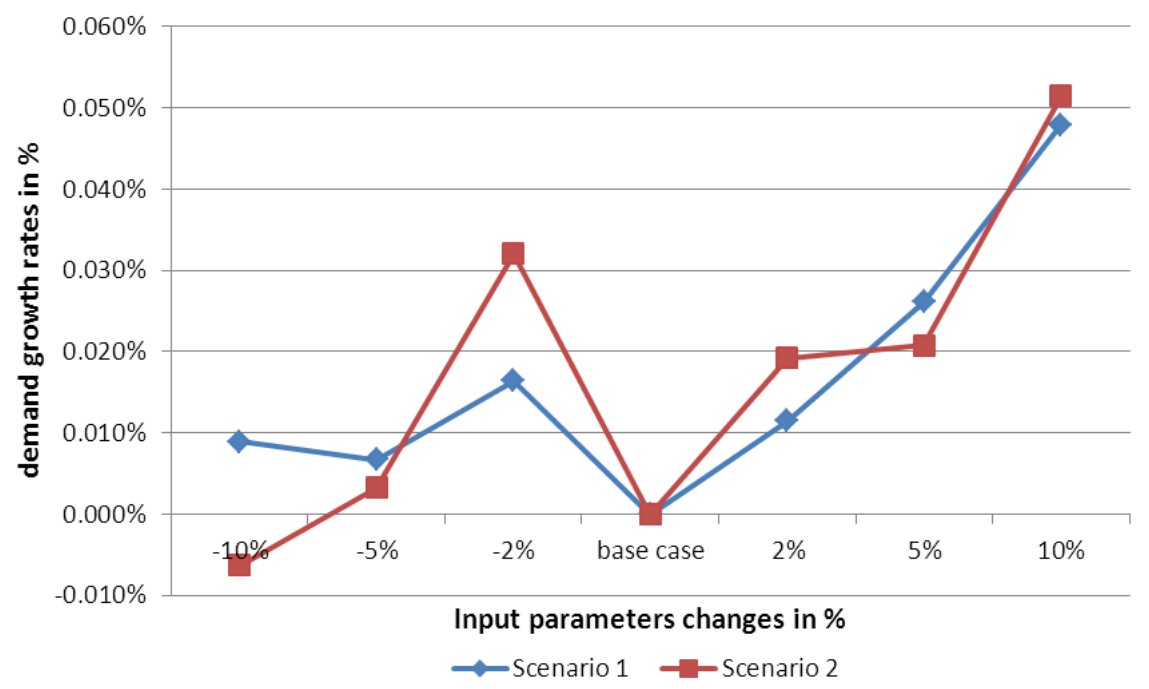

Figure 2: Simulation of WR emergency demand when decreasing and increasing population in two age groups.

\section{DISCUSSION AND CONCLUSIONS}

This study identified the relationship between the number of emergency hospital visits registered for the WR population and three exogenous variables: the total population of the WR, the percentage share of $0-4$ year olds and the percentage share of elderly persons (60+). Based on this relationship, the MC simulation model was developed to forecast the number of emergency presentations to be expected in the WR region. The results are consistent with the available empirical evidence. The sudden changes in population demography were correctly simulated. The errors in emergency patient volume predictions reported in other studies (up to $\pm 11 \%$; cf. Jones et al. 2008) indicate that the predictions are accurate and that the combination of MC simulation and the relatively simple regression model is a reasonable approach. However, further research that would more closely link the demographic trends with regional emergency calls is necessary. When searching for a more accurate model, larger complexity must not necessarily be considered because the input data have to be straightforwardly collected from easily accessible sources (e.g., $\mathrm{CSO}$ webpages). However, the observed differences suggest that the incorporation of exogenous variables is important to the formulation of credible predictions. Furthermore, it might be relevant to connect the demographic changes in the districts with the EMS demand level estimated for the entire region and each particular district separately, which might enable NFZ planners to determine the consequences of the ongoing and observable local population changes for the hospital emergency service needs.

The major strength of the suggested approach lies in the ability to integrate data from different sources (CSO population data and NFZ acute services data) and address 'what-if' policy scenarios by simulating the potential future volume of EMS visits via the observation of actual changes in population 


\section{Mielczarek}

structure. The value of this method lies in the provision of the estimates for the 'what-if' scenarios related to the prognosis of changing acute demand.

The primary limitation of the present study is the limited confidence inspired by past NFZ registries. The ambiguous information on the emergency visits recorded during the first years after the establishment of the NFZ may affect the credibility of the results. Furthermore, the model may only be used to forecast the level of hospital emergency demand for the subsequent year. Long-term analyses would require more careful and thorough identification of the predictors of emergency demand.

The application of simulation modeling to the forecasting of EMS calls originating in different geographical regions might enable the NFZ decision-makers to accurately plan future supply. The regional health authorities may benefit from emergency demand forecast models when beginning contract negotiations and allocating healthcare expenditures in accordance with population needs. Astolfi et al. (2012) compared 25 health expenditure forecast models developed for policy analysis in OECD countries. They identified 7 groups of health-expenditure drivers, with demographic factors forming one group. These researchers also observed that all of the reviewed models accounted for demographic shifts in the population.

Cardoso et al. (2012) described an approach that combined an analytical model with MC simulation to predict the annual demand for long-term care. The authors integrated the stochastic simulation model with a scenario analysis based on a Markov cycle tree structure. The model predicted the number of individuals who would require long-term care during 2010-2015 and the volume of resources required to cover the predicted demand. The authors concluded that this type of integrated approach was a reasonable choice and proved that such a model provides valuable information for policy planners. The problem described in this paper differs from the study reported by Cardoso et al (2012). The demand for EMS services is not constrained by the supply level because emergency services are provided without limits for every patient who requires medical assistance. However, the credible demand predictions might enable the NFZ decision-makers to accurately plan future supply and improve the equity of emergency health services access in the entire region.

The main objective of the next part of our study will be to assess the volume of services to be contracted by NFZ in EDs to satisfy the next-year demand for EMS treatment at the regional level. In particular, we plan to use discrete event simulation to forecast the expected type, volume and cost of medical procedures provided to patients in emergency departments in the WR to cover the predicted demand.

\section{ACKNOWLEDGMENTS}

This project is financed by a grant from the National Science Centre based on decision DEC2011/01/B/HS4/ "Simulation methods and computer simulation models in healthcare management".

\section{REFERENCES}

Aldrich, C. A., J. C. Hisserich, and L. B. Lave. 1971. "An Analysis of the Demand for Emergency Ambulance Service in an Urban Area." American Journal of Public Health 61(6):1156-1169.

Astolfi, R., L. Lorenzoni, and J. Oderkirk, 2012. „Informing Policy Makers About Future Health Spending: A Comparative Analysis of Forecasting Methods in OECD Countries." Health Policy 107:1-10.

Batal, H., J. Tench, S. McMillan, J. Adams, and P. S. Mehler. 2001. "Predicting Patient Visits to an Urgent Care Clinic Using Calendar Variables." Academic Emergency Medicine (1):48-53.

Boyle, J., M. Jessup, J. Crilly, D. Green, J. Lind, M. Wallis, P. Miller, and G. Fitzgerald G. 2012. "Predicting Emergency Department Admissions." Emergency Medical Journal 29(5):358-365.

Cardoso, T., M. D. Oliveira, A. Barbosa-Povoa, and S. Nickel. 2012. "Modeling the Demand for Longterm Care Services Under Uncertain Information." Health Care Management Science 15:385-412.

Central Statistical Office Reports and Statistics. Available http://www.stat.gov.pl/gus [Accessed March 2013] 
Chu, K., A. Brown, and W. Lukin. 2009. "Local Trends in Emergency Department Attendances by Older Patients in an Ageing National Population." Australian Health Review 33(1):117-123.

Diaz-Hierro, J., J. J. Martin Martin, A. Vilches Arenas, M. P. Lopez del Amo Gonzales, J. M. Paton Arevalo, and C. Varo Gonzalez. 2012. "Evaluation of Time-Series Models for Forecasting Demand for Emergency Health Care Services." Emergencias 24:181-188.

Diaz, J., J. C. Alberdi, M. S. Pajares, L. Pharm, C. Lopez, R. Lopez, M. B. Lage, and A. Otero. 2001. "A Model for Forecasting Emergency Hospital Admissions: Effect of Environmental Variables." Journal of Environmental Health 64(3):9-15.

Champion, R., L. D. Kinsman, G. A. Lee, K. A. Masman, E. A. May, T. M. Mills, M. D. Taylor, P. R. Thomas, and R. J. Williams. 2007. "Forecasting Emergency Departments Presentations." Australian Health Review 31(1):83-90.

Channouf, N., P. L'Ecuyer, A. Ingolfsson, and A. N. Avramidis. 2007. "The Application of Forecasting Techniques to Modeling Emergency Medical System Calls in Calgary, Alberta." Health Care Management Science 10:25-4.

Jones, S. S., A. Thomas, R. S. Evans, S. J. Welch, P. J. Haug, and G. L. Snow. 2008. "Forecasting Daily Patient Volumes in the Emergency Department." Academic Emergency Medicine, 15(2):159-170.

Kamenetzky, R. D., L. J. Shuman, and H. Wolfe. 1982. "Estimating Need and Demand for Prehospital Care." Operations Research 30(6):1148-1167.

Kvalseth, T. O., and J. M. Deems. 1979. "Statistical Models of the Demand for Emergency Medical Services in an Urban Area." American Journal of Public Health 69(3):250-255.

Lowthian, J. A., D. J. Jolley, A. J. Curtis, A. Currel, P. A. Cameron, J. U. Stoelwinder, and J. J. McNeil. 2011. "The Challenges of Population Ageing: Accelerating Demand for Emergency Ambulance Services by Older Patients, 1995-2015.” Medical Journal of Australia 194(11):574-578.

Lowthian, J. A., P. A. Cameron, J. U. Stoelwinder, A. Curtis, A. Currel, M. W. Cooke, and J. J. McNeil. 2011. "Increasing Utilization of Emergency Ambulances." Australian Health Review 35:63-69.

McCarthy, M. L., S. L. Zeger, R. Ding, D. Aronsky, N. R. Hoot, and G. D. Kelen. 2008. "The Challenge of Predicting Demand for Emergency Department Services." Academic Emergency Medicine 15(4):337-346.

McConnel, Ch. E, and R. W. Wilson. 1998. "The Demand for Prehospital Emergency Services in an Aging society." Social Science \& Medicine 46(8):1027-1031.

Mielczarek, B., and J. Uziałko-Mydlikowska. 2012a. "Application of Computer Simulation Modeling in the Health Care Sector: a Survey." Simulation. Transactions of the Society for Modeling and Simulation International 88(2):197-216.

Mielczarek, B., and J. Uziałko-Mydlikowska. 2012b. "Using Simulation to Forecast the Demand for Hospital Emergency Services at the Regional Level.” In: Proceedings of the 2012 Winter Simulation Conference, edited by C. Laroque, J. Himmelspach, R. Pasupathy, O. Rose, and A. M. Uhrmacher. Piscataway, New Jersey: Institute of Electrical and Electronics Engineers, Inc.

Ong, M. E. H., K. K. Ho, T. P. Tan, S. K. Koh, Z. Almuthar, J. Overton, and S. H. Lim. 2009. "Using Demand Analysis and System Status Management for Predicting ED Attendances and Rostering." American Journal of Emergency Medicine 27:16-22.

Setzler, H., C. Saydam, and S. Park. 2009. "EMS Call Volume Predictions: A Comparative Study." Computers \& Operations Research 36:1843-1851.

\section{AUTHOR BIOGRAPHY}

BOŻENA MIELCZAREK is an assistant professor of Management Science at Wrocław University of Technology, Poland. She received an MSc in Management Science and a $\mathrm{PhD}$ in Economics from Wrocław University of Technology. Her research interests include simulation modeling, health-service research, decision support and risk analysis. Her email address is bozena.mielczarek@pwr.wroc.pl. 